\title{
Eficacia de la maca fresca (Lepidium meyenii walp) en el incremento del rendimiento físico de deportistas en altura
}

\author{
Gerardo Ronceros ${ }^{1,2}$, Willy Ramos ${ }^{1,2}$, Fausto Garmendia ${ }^{1}$, \\ Jorge Arroyo ${ }^{1}$, Julio Gutiérrez ${ }^{2}$
}

Resumen

Palabras clave
Objetivo: Determinar el efecto de la maca fresca sobre el rendimiento físico de deportistas en la altura. Diseño: Estudio experimental, prospectivo y comparativo. Materiales y Métodos: La muestra estuvo constituida por 10 deportistas de altura, quienes fueron sometidos a actividad física para medir el consumo máximo de oxígeno $\left(\mathrm{VMO}_{2}\right.$ ) basal; luego, se les administró durante 60 días 1500 mg/día de maca fresca en presentación micropulverizado y se determinó el consumo máximo de oxígeno. En cada evaluación se realizó dosaje de transaminasa glutámico oxalacética (TGO), transaminasa glutámico pirúvica (TGP) y creatinina sérica. Resultados: En la evaluación basal, se encontró que $\mathrm{VMO}_{2} 2 \mathrm{~mL} / \mathrm{kg} / \mathrm{min}$. Al realizar la evaluación luego de 60 días del consumo de la maca fresca, se encontró que la media del $\mathrm{VMO}_{2}$ fue $57,75 \mathrm{~mL} / \mathrm{kg} / \mathrm{min}$ ( $p=0,001)$. Se demostró un incremento del rendimiento físico de los deportistas en promedio de 10,3\%. En todos los casos, los valores de TGO, TGP y creatinina sérica se mantuvieron en el rango de normalidad y no se presentaron reacciones adversas. Conclusiones: La maca fresca es un producto con actividad energética, que permite el incremento del rendimiento físico de deportistas en la altura a una dosis de 1500 mg/día, durante un período de administración de 60 días.

Lepidium meyenii walp; esfuerzo físico; deportes; altitud; consumo de oxígeno.
Efficacy of fresh maca (Lepidium meyenii walp) in the sportsmen increment in physical performance at high altitude

\section{Abstract}

Objective: To establish the effect of fresh maca (Lepidum meyenil walp) on the sportsmen physical performance at high altitude. Design: Comparative, prospective, and experimental study. Materials and Methods: Ten high altitude sportsmen constituted the sample. We first measured the maximum oxygen consumption under physical activity. Then they received $1500 \mathrm{mg}$ of fresh maca daily during 60 days and a second evaluation was performed under similar conditions. The maximum oxygen intake and performance were determined and subjects were monitored for a 30 days period. Dosage of transaminases

1 Instituto de Investigaciones Clínicas. Facultad de Medicina, UNMSM. Lima, Perú.

2 Laboratorios Schüler. Lima, Perú.
ALAT, ASAT, and serum creatinine were done during each evaluation. Results: In basal conditions the velocity average in the treadmill was $15,46 \mathrm{~km} / \mathrm{h}$ and the maximum oxygen intake average was $52,32 \mathrm{~mL} / \mathrm{kg} / \mathrm{min}$. In the second evaluation following the fresh maca consume the average of the maximum velocity raised to $16,89 \mathrm{~km} / \mathrm{h}$ and the maximum oxygen intake was $57,75 \mathrm{~mL} / \mathrm{kg} / \mathrm{min}$, which means a 10,3\% increase in physical performance. In all cases, the ALAT, ASAT, and serum creatinine values were normal. Conclusions: Fresh maca revealed energizing properties that increase the sportsmen physical performance at high altitude.

Keywords: Lepidium meyenil walp; exertion; sports; altitude; oxygen consumption.

\section{INTRODUCCIÓN}

La maca es la raíz de la planta Lepidium meyenii, miembro de la familia Brassicacae, 
conocida y usada desde la época del imperio incaico. Crece en los andes centrales del Perú, entre $\operatorname{los} 3000$ y 4500 metros de altitud, prácticamente restringida a los departamentos de Junín, Pasco, Ancash y Puno (a orillas del Lago Titicaca). El Lepidium meyenii se caracteriza por su crecimiento a temperaturas bajas; así, puede crecer en un rango de $-1,5$ a $12^{\circ} \mathrm{C}$, en suelos húmedos y ácidos con un $\mathrm{pH}$ menor o igual a $5\left({ }^{(1-3)}\right.$. En cuanto a su caracterización química, se ha aislado algunos de sus principios activos, como el derivado benzilado 1,2-dihidro-N_hidroxipiridina, llamado macaridina, las macamidas, N-benzil5-oxo-6E, 8Eoctadecadienamida, N-benzilhexadecanamida, ácido acético, 5-oxo-6E, 8Eácido octadecadienoico y los glucoenolatos $\left({ }^{4}\right)$.

La maca ha sido tradicionalmente empleada como afrodisiaco y para incrementar la fertilidad. Más recientemente, se ha demostrado que mejora la espermatogénesis en ratas mediante el incremento del volumen de semen, aumento del número y motilidad de los espermatozoides $\left({ }^{1,2}\right)$.

Diversos estudios han demostrado su valor nutritivo como suplemento de la alimentación, debido a su alto contenido de carbohidratos (59\%), lípidos $(2.2 \%)$, proteínas $(10,2 \%)$, fibra $(8,5 \%)$, aminoácidos (ácido glutámico, ácido aspártico, serina, glicina, arginina), ácidos grasos saturados, ácidos grasos esenciales (palmítico, linolénico), minerales (hierro y calcio) y esteroles $\left(^{3}\right)$.

Por otra parte, es conocido que, en el deporte, sobre todo en la altura, se requiere mejorar el rendimiento físico y optimizar el consumo de oxígeno, para lo cual existen pocas sustancias de uso lícito que permitan obtener este beneficio. Se señala que la maca posee efecto energético y que los incas usaban esta planta para obtener el vigor para combatir en las batallas y derrotar a sus enemigos $\left({ }^{3,5,6}\right)$.

El aumento del rendimiento físico de los deportistas puede ser medido por diversos métodos, tanto directos como indirectos. El consumo máximo de oxígeno $\left(\mathrm{MVO}_{2}\right)$ es uno de los indicadores más importantes a tener en cuenta cuando se necesita evaluar el rendimiento de los deportistas $\left({ }^{7}\right)$.

En la literatura se señala una diversidad de ecuaciones predictivas del $\mathrm{MVO}_{2}$, utilizadas para tal fin en diferentes variables, como la velocidad máxima alcanzada durante una carrera en la banda sin fin, el porcentaje de la frecuencia cardiaca máxima alcanzada, etc $\left(^{8}\right)$.

De acuerdo a los estudios de María Gonzáles y col., los métodos Ricart y de Pugh son los que menos porcentaje de error presentan con respecto al método directo. Estos valores están comprendidos dentro del rango de error establecido por la literatura cuando se utiliza métodos indirectos. Los resultados obtenidos con la fórmula de Pugh $\left({ }^{9}\right.$ ) son los que tienen el menor porcentaje de error $\left({ }^{10}\right)$, por lo cual es el método indirecto más eficaz para determinar el rendimiento de los deportistas mediante el $\mathrm{MVO}_{2}$.

El presente estudio está planteado para determinar la actividad energética de la maca en deportistas sometidos a ejercicio físico en la altura. Se usa la prueba del consumo máximo de oxígeno $\left(\mathrm{MVO}_{2}\right)$ para objetivar el incremento del rendimiento físico, calculado mediante la fórmula de Pugh.

\section{MATERIALES Y MÉTODOS}

El diseño corresponde a un estudio prospectivo, longitudinal y experimental realizado en el período comprendido entre agosto a diciembre del año 2003 en la ciudad del Cusco, la cual se encuentra a $3400 \mathrm{msnm}$.

La muestra estuvo constituida por deportistas de altura de un equipo de fútbol profesional (Cienciano del Cusco). El muestreo fue de tipo no probabilístico, que incluyó a 10 jugadores de fútbol de dicha institución, quienes aceptaron enrolarse voluntariamente en el estudio y firmaron un consentimiento informado. Durante 
la realización del estudio, se mantuvo una rutina física constante, de modo que ésta no interfiriera con el rendimiento físico.

Los deportistas fueron sometidos a actividad física antes de ingresar al estudio, de manera de medir el consumo máximo de oxígeno basal. Para esto, se utilizó una máquina con una faja sin fin, que midió la velocidad máxima desarrollada en un tiempo determinado (12 minutos), siendo este dato empleado en la fórmula de Pugh.

Luego, durante 60 días recibieron tres cápsulas de concentrado de maca fresca (procesada sin ser sometida a desecación), en presentación micropulverizada, conteniendo 500 mg cada una (1 $500 \mathrm{mg}$ por día). Posteriormente, fueron sometidos nuevamente a actividad física similar a la inicial, determinándose la velocidad máxima, el consumo de oxígeno, el rendimiento y se realizó nuevamente dosaje de TGO, TGP y creatinina sérica.

El estudio incluyó un período de seguimiento de 30 días luego de finalizada la administración de maca fresca, con la finalidad de evaluar posibles efectos adversos que se presentaran luego de dejar de administrar el producto. Asimismo, se determinó los niveles de TGO,

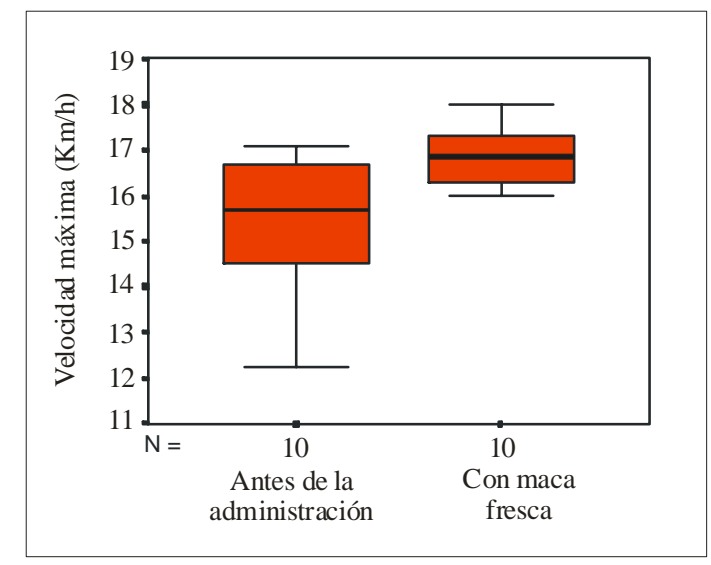

Figura 1. Velocidad máxima (en km/h) de los deportistas antes y después de la administración de maca fresca.
TGP y creatinina sérica. El rendimiento de los deportistas en la altura fue evaluado de manera indirecta mediante la determinación del consumo máximo de oxígeno $\left(\mathrm{MVO}_{2}\right)$. Para esto, se utilizó el método de Pugh $\left({ }^{10}\right)$.

$\mathrm{MVO}_{2}(\mathrm{~mL} / \mathrm{kg} \mathrm{x} \mathrm{min})=(3,656$ x V $)-3,99$

donde: $\mathrm{V}=$ velocidad en $\mathrm{km} / \mathrm{h}$

Para el procesamiento de los datos, se utilizó el programa estadístico SPSS versión 11,0 y, para el análisis estadístico, la prueba de Wilcoxon.

\section{RESULTADOS}

En la evaluación basal, se encontró que la media de la velocidad obtenida en la faja sin fin fue de $15,46 \mathrm{~km} / \mathrm{h}$ y la media del consumo máximo de oxígeno $\left(\mathrm{VMO}_{2}\right)$ fue $52,32 \pm 5,68$ $\mathrm{mL} / \mathrm{kg} / \mathrm{min}$ (Figuras 1 y 2, Tabla 1 ).

En la evaluación basal, los valores de TGO fueron $17,8 \pm 7,02 \mathrm{U} / \mathrm{L}$ y para la TGP $18,4 \pm$ $5,1 \mathrm{U} / \mathrm{L}$. La media para los valores de creatinina sérica fue $0,79 \pm 8,22-2 \mathrm{mg} / \mathrm{dL}$; ningún deportista excedió los valores normales.

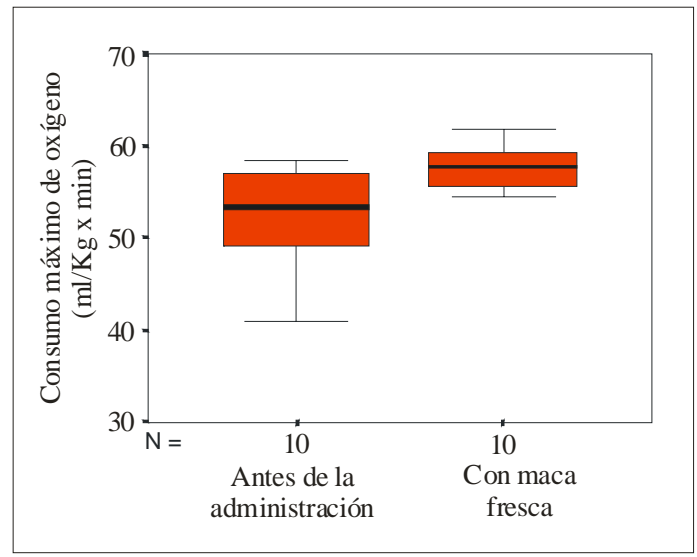

Figura 2. Consumo máximo de oxígeno (en $\mathrm{mL} / \mathrm{kg} / \mathrm{min}$ ) antes y después de la administración de maca fresca. 
Tabla 1. Consumo máximo de oxígeno (en $\mathrm{mL} / \mathrm{kg} / \mathrm{min}$ ) antes y después de la administración de maca fresca.

\begin{tabular}{lcc}
\hline & \multicolumn{2}{c}{ Consumo máximo de oxígeno/kg } \\
\cline { 2 - 3 } & sin maca fresca & con maca fresca \\
\hline Media & 52,32 & 57,75 \\
Desviación estándar & 5,68 & 2,47 \\
Varianza & 32,34 & 2,47 \\
Rango & 17,72 & 7,31 \\
Mínimo & 40,80 & 54,50 \\
Máximo & 58,52 & 61,81 \\
\hline
\end{tabular}

Al realizarse la evaluación luego de 60 días del consumo de la maca fresca con una dosis de $1500 \mathrm{mg}$ diarios, se encontró un incremento significativo de la velocidad máxima y del consumo máximo de oxígeno $\left(\mathrm{VMO}_{2}\right)$ de los deportistas (Figuras 1 y 2). La media de la velocidad máxima se incrementó a $16,89 \mathrm{~km} / \mathrm{h}$ y para el $\mathrm{VMO}_{2}$ la media fue $57,75 \pm 2,47 \mathrm{~mL} /$ $\mathrm{kg} / \mathrm{min}$ (Tabla 1 ).

Se encontró diferencia estadísticamente significativa entre las medias de las velocidades máximas alcanzadas antes y después del consumo de maca fresca, a favor del consumo de maca fresca $(p=0,001)$. De esta manera, el rendimiento físico de los deportistas se incrementó en promedio 10,3\% luego de la administración. Los valores máximos para el incremento del consumo de oxígeno fueron $33,6 \%$ para un deportista, mientras que el valor mínimo fue $5,6 \%$.

Asimismo, la media de valores de TGO fue $18,01 \pm 1,11 \mathrm{U} / \mathrm{L}$, mientras que para la TGP fue $18,2 \pm 0,87 \mathrm{U} / \mathrm{L}$. La media para los valores de creatinina sérica fue $0,78 \pm 7,33-2 \mathrm{mg} /$ $\mathrm{dL}$. No se presentaron reacciones adversas durante el período de administración de maca fresca.

Se encontró diferencia estadísticamente significativa para el consumo máximo de oxígeno antes y después del consumo de maca fresca $(p=0,001)$ a favor de la administración de maca.
Ningún deportista excedió los valores normales de transaminasas y creatinina sérica durante el período de seguimiento.

\section{DISCUSIÓN}

Los pobladores de los Andes peruanos atribuyen a la maca un efecto energético. El presente es el primer estudio que demuestra científicamente que la maca incrementa el rendimiento físico en la altura. Es así que, se consiguió incrementar el rendimiento físico en la muestra estudiada en $10,3 \%$, a pesar de tratarse de deportistas en constante actividad física, entrenados para la competencia de alto nivel.

De acuerdo a esto, creemos que si la maca fresca fuera empleada en la población general no vinculada al deporte, el beneficio en el rendimiento físico debería ser mayor. Esto se sustenta en que en los deportistas, debido a que se encuentran en constante trabajo físico, es difícil conseguir grandes incrementos en el rendimiento físico, lo cual es factible en las personas que no se dedican al deporte profesional.

El beneficio otorgado por la maca fresca puede ser mucho mayor en algunos deportistas, registrándose un incremento hasta de 33,6\%. Esto podría explicarse debido a que la respuesta a la administración es distinta en cada deportista, pero el incremento del rendimiento físico se presentó en todos ellos.

De igual modo, es factible que el incremento del rendimiento físico sea mayor a nivel del mar, por lo que es necesaria la realización de estudios de maca fresca para demostrar esta hipótesis.

Los resultados de esta investigación muestran que la maca fresca es un producto seguro, debido a que no se presentaron reacciones adversas durante el tiempo de tratamiento ni durante el período de seguimiento. Ello se evidenció también en los valores de transaminasas y creatinina sérica, que no superaron los valores normales en los deportistas. 
Creemos necesaria la realización de nuevos estudios que incluyan mayor muestra y que confirmen los hallazgos aquí descritos, permitiendo la validación de este producto y su uso en el deporte.

\section{REFERENCIAS BIBLIOGRÁFICAS}

1. Gonzales GF, Cordova A, Gonzales C, Chung A, Vega $\mathrm{K}$, Villena A. Lepidium meyenii (maca) improved semen parameters in adult men. Asian J Androl. 2001;3:301-3.

2. Gonzales GF, Ruiz A, Gonzales C, Villegas L, Cordova A. Effect of Lepidium meyenii (maca) roots on spermatogenesis of male rats. Asian J Androl. 2001;3:2313.

3. Hermann M, Heller J. Andean roots and tubers: Ahipa, arracacha, maca, yacon. Rome: International Plant Genetic Resources Institute; 1997 p. 175-194.

4. Muhamma I, Zhao J, Dunbar C, Khan IA. Constituents of Lepidium meyenii "maca". Phytochemistry. 2002;59:10510.

5. Piacente S, Carbone V, Plaza A, Zampelli A, Pizza C. Investigations of the tuber constitutiens of maca (Lepidium meyenii walp). J Agric Food Chem. 2002;50:5621-5.

6. Cicero AFG, Piacente S, Plaza A, Sala E, Arletti R, Piza C. Hexanic maca extract improves rat sexual performance more effectively than methanolic and chloroformic maca extracts. Andrologia. 2002;34:177-9.
7. Ferrero JA, Fernández VA. Consumo de oxígeno: concepto, bases fisiológicas y aplicaciones. En: López Chicharro J, Fernández VA. Fisiología del ejercicio. Madrid: Editorial Médica Panamericana; 1995. p. 209-18.

8. Rodríguez GF. Valoración funcional de la capacidad de rendimiento físico. En: González Gallego. Fisiología de la actividad física y el deporte. Nueva York: Interamericana Mc Graw Hill; 1992. p. 237-74.

9. Pugh L. Oxygen intake in track and treadmill running with observations on the effect of air resistance. J Physiol. 1970;207:283.

10. González ME, Castellanos P, Almenares E, Sánchez A, López A. Determinación indirecta del máximo consumo de oxígeno. Estudio comparativo de tres métodos. efdeportes.com [serial en la Internet]. 2002 [citado 2006 Ene 15];8(46):[aprox. 3 p.]. Disponible en: http:// www.efdeportes.com/efd46/maxcon.htm

Manuscrito recibido el 15 de noviembre de 2005 y aceptado para publicación el 20 diciembre de 2005.

Correspondencia: Dr. Gerardo Ronceros Medrano Hospital Nacional Dos de Mayo Instituto de Investigaciones Clínicas, UNMSM. Av. Grau cuadra 13, Parque Historia de la Medicina Peruana Lima 1, Perú

Correo-e: investcl@yahoo.com 